\title{
Isolated cardiac tamponade following blunt trauma in an infant
}

\author{
Philip Maykowski, MPH ${ }^{\mathrm{a}}$, Jae-O. Bae, MD ${ }^{\mathrm{b}}$, David M. Notrica, MD ${ }^{\mathrm{a}, \mathrm{b}, \mathrm{c} \text {,* }}$ \\ ${ }^{\text {a } U n i v e r s i t y ~ o f ~ A r i z o n a ~ C o l l e g e ~ o f ~ M e d i c i n e ~-~ P h o e n i x, ~ P h o e n i x, ~ A Z, ~ U S A ~}$ \\ b Department of Surgery, Phoenix Children's Hospital, Phoenix, AZ, USA \\ ${ }^{\mathrm{c}}$ Department of Surgery, Mayo Clinic, Phoenix, AZ, USA
}

\section{A R T I C L E I N F O}

\section{Keywords:}

Cardiac tamponade

Blunt trauma

Infant

\begin{abstract}
A B S T R A C T
This is a case of a 6-month old female with no prior medical history who presented to the emergency department following a high-speed motor collision with ejection of an improperly installed car seat. The patient was hemodynamically unstable with tachycardia, poor perfusion, and marks from the car seat. A focused assessment with sonography in trauma (FAST) ultrasound exam revealed no intraabdominal fluid, but evidence of cardiac tamponade which was confirmed by chest CT angiography. No cardiac or great vessel injury was present. A pericardial window was performed using a subxiphoid approach and $150 \mathrm{mLs}$ of blood was drained resulting in immediate hemodynamic stabilization. The patient continued to improve from a cardiac stand point but her hospital course was complicated by iatrogenic right arm ischemia due to arterial line attempt. The patient was discharged after a 12-day hospital stay.
\end{abstract}

\section{Introduction}

Blunt trauma to the chest in infants is a known cause of myocardial contusion, cardiac rupture, and acute valvular disruption. Detecting these injuries can be difficult, but failure to diagnose carries significant morbidity and/or mortality [1]. By contrast, isolated cardiac tamponade from blunt trauma without structural cardiac injury is a rare complication of blunt trauma [2]. This unexpected clinical scenario of blunt trauma and cardiac tamponade, particularly in pediatric patients, can present with subtle symptoms or even be initially asymptomatic [3-5] Guidelines on detection, management, and outcomes of isolated pericardial tamponade after blunt trauma are sparse in pediatrics with seven associated case reports in children of any age and none under 1 year of age. We present a case of a 6-month old involved in a high-speed motor vehicle accident with a pericardial effusion without other associated cardiac injuries.

\section{Case report}

The patient was a 6-month old female with no past medical history, normal prenatal course, and appropriate development who presented to the emergency department hemodynamically unstable for unclear reasons following a high-speed motor collision with ejection of an improperly installed car seat. The collision was a frontal impact motor vehicle collision with severe front-end damage. The child had remained in the car seat which was found distant from the crash. The car seat had not been secured to the car, thus both the car seat and child were ejected out of the car upon impact.

\subsection{Emergency department course}

On arrival she had a Glasgow comma scale (GCS) of 12, tachycardia $(\geq 200 \mathrm{bpm}$ ) strap marks on the right chest and right upper quadrant of abdomen, and right clavicular bruising without crepitus. An airway was secured by placing an endotracheal tube and the patient was resuscitated with a normal saline bolus. Despite this, the patient's perfusion continued to deteriorate and an empiric emergent chest tube was then placed. FAST ultrasound exam indicated fluid present around the heart, but no intraabdominal fluid and the venous blood gases showed continued acidosis $(\mathrm{pH}=6.94)$. The patient's perfusion and hemodynamic status improved temporarily following the emergent chest tube placement and transfusion. At that time, she was transferred to CT (Fig. 1) to evaluate for aortic injury prior to committing to a subxiphoid pericardial window in the supine position. However, direct transfer to the OR might have been equally as appropriate in retrospect.

\footnotetext{
* Corresponding author. Department of Surgery, Phoenix Children's Hospital, 1919 East Thomas Road, Phoenix, AZ, 85016, USA.

E-mail address: dnotrica@phoenixchildrens.com (D.M. Notrica).
} 


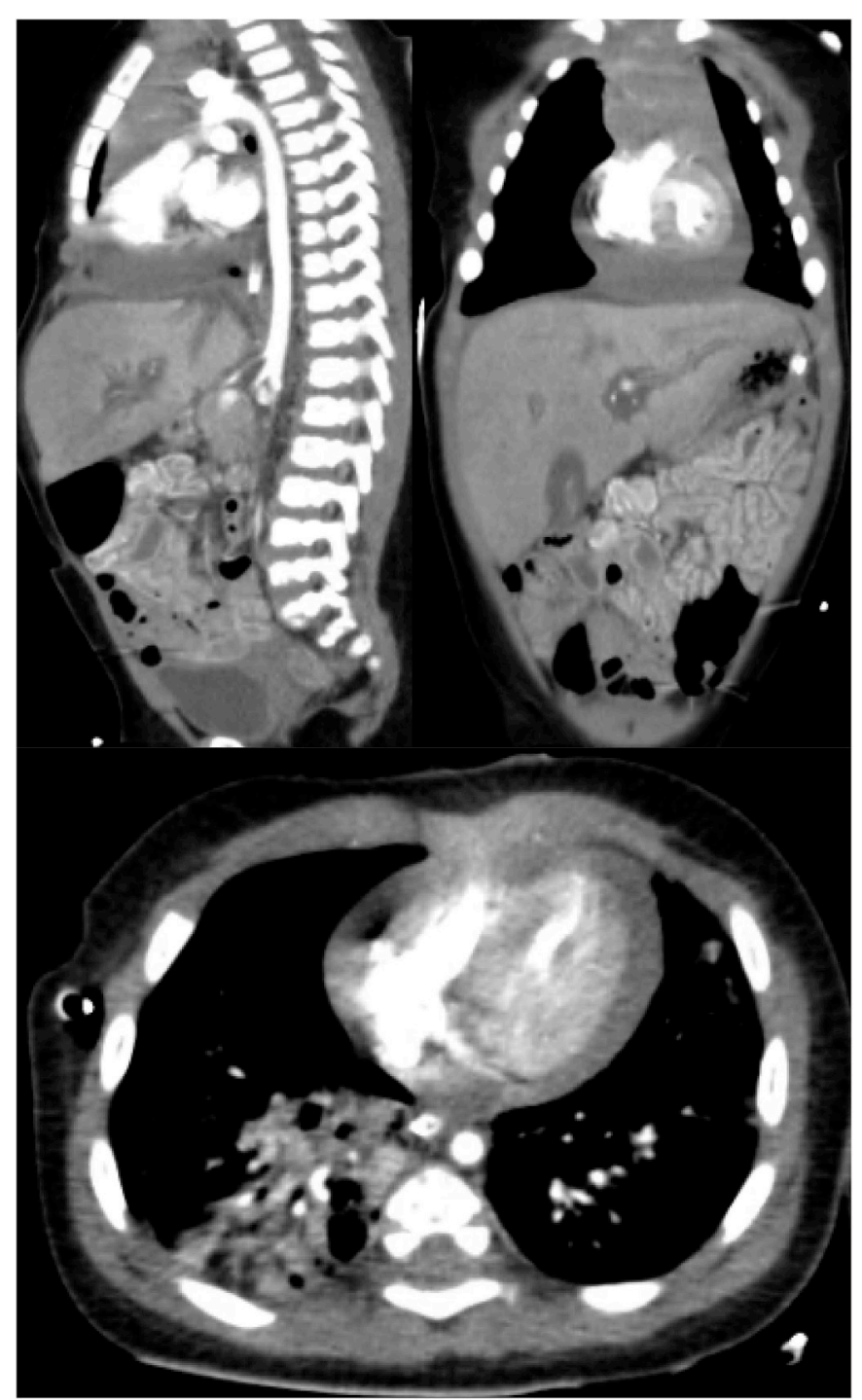

Fig. 1. Sagittal, coronal, and axial CT of the chest and abdomen demonstrating a right pneumothorax, hemopericardium with possible compression for the right heart, fractures on the 6th and 7th ribs, and diffuse shock bowel. Despite the pericardial effusion, the heart was noted to be normal in size, without associated visible injury.

\subsection{Surgical course}

The chest CT confirmed cardiac tamponade without concurrent structural cardiac or aortic injury, however, she then began to lose hemodynamic stability, required further transfusions, and was transferred urgently to the OR. A pericardial window was performed using a subxiphoid approach. Upon initial access to the mediastinum, a collection of dark purple fluid was noted around the heart. The tense pericardium was incised and $150 \mathrm{mLs}$ of fluid was drained from the area resulting in immediate hemodynamic stabilization. Hemostasis in the pericardium was achieved after a transfusion of FFP and platelets. No ongoing hemorrhage was noted and the remainder of the heart appeared uninjured. The right chest tube was replaced in the OR and a 19-French Blake drain was placed in the right upper quadrant of the abdominal wall through the previously made subxiphoid incision to allow for continued drainage of the pericardium as needed. Estimated blood loss was $450 \mathrm{~mL}$ and post-operative blood gases demonstrated a $\mathrm{pH}$ of 7.19 with $\mathrm{CO}_{2}$ of $57 \mathrm{mmHg}, \mathrm{pO}_{2}$ of $125 \mathrm{mmHg}$ and bicarbonate of $21 \mathrm{mmol} / \mathrm{L}$.

\subsection{Post-operative course}

Her course was complicated by right arm and hand ischemia noted immediately after the pericardial window surgery. This was believed to be from an unsuccessful brachial artery line attempt. Hand surgery noted two small puncture wounds in the brachial region as well as ecchymosis and abrasion on the distal forearm, which could explain the acute arm/hand ischemia. The intraoperative arterial line had been radial. She underwent a proximal radial artery and brachial artery thrombectomy with improvement in arm perfusion. Hand fasciotomies and carpal tunnel release were also done. She later required debridement of the forearm soft tissues. The patient was extubated on hospital day 5 and discharged on hospital day 12. The patient recovered well from a cardiothoracic standpoint. On follow up she required further debridement of her arm due to necrosis from the original ischemia and as well as loss of several finger tips. She recovered from this following a short hospitalization and IV antibiotics.

\section{Discussion}

Unintentional injury remains one of the leading cause of pediatric mortality in the United states with MVCs accounting for the majority of cases [6,7]. Specifically, chest trauma accounts of approximately $25 \%$ of trauma mortality with blunt trauma being the most common mechanism in children [8]. In the case of blunt cardiac injury, the presentations may be subtle, including tachycardia, and is often hard to distinguish with co-occurring traumatic morbidity which makes early detection difficult [9].

Cardiac tamponade is rare following blunt trauma and never previously reported in infants. Young children present with subtle hemodynamic signs and mental status findings which can be especially difficult to assess in children [10]. Seven prior cases of pericardial tamponade in children after blunt injury have been reported [4,5,9-13]. These cases included ages 21-months to 17 years. Four of the seven patients presented with associated cardiac injuries, three with ventricular rupture and one with an SVC tear, and two of the seven patients did not survive their injuries.

Our patient presented with non-specific tachycardia and altered mental status. There were not obvious signs of cardiac tamponade such as neck vein distention. However, the hemodynamic instability and worsening labs despite resuscitation warranted expedited FAST exam. The inclusion of FAST exams in routine trauma care may or may not have benefit for stable patients, but remains essential in the evaluation of unstable children. Given the level of instability of our patient the FAST exam was essential to making an early diagnosis in the setting of instability after blunt trauma and directed care.

\section{Conclusions}

Our patient represents an uncommon scenario of hemodynamic instability in an infant caused by cardiac tamponade without major cardiac injury after blunt trauma. This infant is the youngest reported case in the current literature. Despite an absence of classic clinical features, cardiac tamponade was successfully detected and appropriately treated with good outcome. Use of FAST exam in this unstable infant resulted in early treatment and most likely improved the outcome.

\section{Declarations of interest}

None.

\section{Funding}

This research did not receive any specific grant from funding agencies in the public, commercial, or not-for-profit sectors. 


\section{Conflicts of interest}

The following authors have no financial disclosures: PM, JB, DN.

\section{Patient consent}

Consent to publish the case report was not obtained. This report does not contain any personal information that could lead to the identification of the patient.

\section{Authorship}

All authors attest that they meet the current ICMJE criteria for Authorship.

\section{References}

[1] Marcolini EG, Keegan J. Blunt cardiac injury. Emerg Med Clin N Am 2015;33(3): 519-27. https://doi.org/10.1016/j.emc.2015.04.003.

[2] Fitzgerald M, Spencer J, Johnson F, Marasco S, Atkin C, Kossmann T. Definitive management of acute cardiac tamponade secondary to blunt trauma. Emerg Med Australasia (EMA) 2005;17(5-6):494-9. https://doi.org/10.1111/j.17426723.2005.00782.x.

[3] Takamatsu J. Pericardial injury with cardiac tamponade and bleeding from the pericardium confirmed using contrast-enhanced computed tomography: a case report. Surg Case Rep 2019;5(1). https://doi.org/10.1186/s40792-019-0584-y. 32.
[4] Campo dell' Orto M, Kratz T, Wild C, et al. Pre-hospital ultrasound detects pericardial tamponade in young patients with occult blunt trauma: time for preparation? Case report and review of literature. Clin Res Cardiol 2014;103(5): 409-11. https://doi.org/10.1007/s00392-014-0661-y.

[5] Murillo CA, Owens-Stovall SK, Kim S, Thomas RP, Chung DH. Delayed cardiac tamponade after blunt chest trauma in a child. J Trauma 2002;52(3):573-5. https://doi.org/10.1097/00005373-200203000-00029.

[6] National Center for Injury Prevention and Control. CDC childhood injury report: pattern of unintentional injuries among 0-19 year olds in the United States. Accessed July 29, 2019, https://www.cdc.gov/safechild/pdf/cdc-childhoodinjury. pdf). Centers for Disease Control and Prevention. [Accessed 23 December 2015].

[7] National Center for Injury Prevention and Control. 10 leading causes of death by age group, United States - 2017. Accessed, https://www.cdc. gov/injury/images/lccharts/leading_causes_of_death by_age_group_2017_1100w850h.jpg). Centers for Disease Control and Prevention. [Accessed 29 July 2019].

[8] Pauze D, Pauzé DK. Emergency management of blunt chest trauma in children: an evidence based-approach. Pediatr Emerg Med Pract 2013;10:1-22.

[9] Kanchan T, Menezes RG, Acharya PB, Monteiro FN. Blunt trauma to the chest-a case of delayed cardiac rupture. J Forensic Leg Med 2012;19(1):46-7.

[10] Arciniegas Rodriguez S, Zitek T, Sterett R, Nelson DG. Occult pericardial tamponade presenting with altered mental status. Pediatr Emerg Care 2018;34(5): e93-4. https://doi.org/10.1097/PEC.0000000000000878.

[11] Mozzetti MD, Devin JB, Susselman MS, Lammert GR, Olshaker JS. A pediatric survivor of left ventricular rupture after blunt chest trauma. Ann Emerg Med 1990; 19(4):386-9. https://doi.org/10.1016/s0196-0644(05)82341-8.

[12] Bowers P, Harris P, Truesdell S, Stewart S. Delayed hemopericardium and cardiac tamponade after unrecognized chest trauma. Pediatr Emerg Care 1994;10(4): 222-4.

[13] Tabansi PN, Otaigbe BE. Late onset hemopericardium with cardiac tamponade from minor blunt chest trauma - a case report. Clin Case Rep 2015;3(4):247-50. https://doi.org/10.1002/ccr3.216. 